Research Paper

\title{
Intensity-modulated radiotherapy provides better quality of life than two-dimensional conventional radiotherapy for patients with stage II nasopharyngeal carcinoma
}

\author{
Xin-Bin Pan ${ }^{1}$, Shi-Ting Huang ${ }^{1}$, Kai-Hua Chen ${ }^{1}$, Yan-Ming Jiang ${ }^{1}$, Jia-Lin Ma ${ }^{1}$, Song \\ $\mathbf{Q u}^{1}$, Ling $\mathrm{Li}^{1}$, Long Chen ${ }^{1}$ and Xiao-Dong Zhu ${ }^{1}$ \\ ${ }^{1}$ Department of Radiation Oncology, Cancer Hospital of Guangxi Medical University, Nanning, Guangxi 530021, P.R. China \\ Correspondence to: Xiao-Dong Zhu, email: zhuxiaodonggx@163.com \\ Keywords: nasopharyngeal carcinoma, quality of life, intensity-modulated radiotherapy, two-dimensional conventional radiotherapy \\ Received: January 02, $2017 \quad$ Accepted: April 03, $2017 \quad$ Published: May 03, 2017 \\ Copyright: Pan et al. This is an open-access article distributed under the terms of the Creative Commons Attribution License 3.0 (CC \\ BY 3.0), which permits unrestricted use, distribution, and reproduction in any medium, provided the original author and source are \\ credited.
}

\section{ABSTRACT}

Two-dimensional conventional radiotherapy (2D-CRT) and intensity-modulated radiotherapy (IMRT) are effective for control of nasopharyngeal carcinoma (NPC). The purpose of this study was to compare the quality of life (QOL) of stage II NPC patients treated with 2D-CRT versus IMRT. We conducted a cross-sectional study of 106 patients with stage II NPC treated with 2 D-CRT $(n=47)$ versus IMRT $(n=59)$ between June 2008 and June 2013. For all subjects, disease-free survival was more than 3 years. QoL was assessed using the European Organization for Research and Treatment of Cancer Quality of Life Questionnaire-Core 30 (EORTC QLQ-C30) questions and the Head and Neck 35 (EORTC QLQ-H\&N35) questions. Patients receiving IMRT with or without concurrent chemotherapy had better outcomes in head and neck related symptoms and general aspects of QoL than those receiving 2D-CRT with or without concurrent chemotherapy. Thus, IMRT improves the QoL of patients with stage II NPC as compared to 2D-CRT.

\section{INTRODUCTION}

Nasopharyngeal carcinoma (NPC) is highly endemic in southern China [1]. Radiotherapy is the primary treatment modality for NPC. Two-dimensional conventional radiotherapy (2D-CRT) has been effective in controlling NPC, but complications to organs at risk resulting from 2D-CRT are severe and lifelong. In the last decade, intensity-modulated radiotherapy (IMRT) has rapidly replaced 2D-CRT due to its technical and dosimetric superiority, and when resources permit, has become the most commonly used radiation technique for NPC.

The incidence of stage II NPC has greatly increased with improvements in diagnosis, and after treatment the 5 -year overall survival is assumed to be $95 \%$ or higher $[2,3]$. The high survival rate makes quality of life $(\mathrm{QoL})$ increasingly important. Previous studies suggested that IMRT improved symptoms and QoL for NPC survivors [4-6]. However, all of these studies were confounded by the interference of chemotherapy [7, 8]. Moreover, while the previous studies treated NPC as a whole group and analyzed
QoL, most patients had advanced loco-regional NPC. Only one randomized controlled trial compared QoL of IMRT versus 2D-CRT in early stage NPC [9]. This trial suggested that IMRT was superior with regard to speech problems and swallowing. However, another randomized controlled trial reported that there was no significant difference in patientreported xerostomia between IMRT and 2D-CRT [10]. Moreover, in both studies the sample size was relatively small, and the follow-up time was only 1 year, and neither provided accurate information regarding the QoL of IMRT versus $2 \mathrm{D}-\mathrm{CRT}$ in early stage NPC.

In developing regions, many patients are treated with 2D-CRT rather than IMRT because they have no access to IMRT or the financial burden of IMRT is too great. Although 2D-CRT provides a similar survival benefit for NPC as IMRT [11, 12], clinicians have begun to pay more attention to QoL. We conducted a cross-sectional study to compare the QoL between IMRT and 2D-CRT in patients with stage II NPC. The result of this study might help clinicians make treatment decisions and provide information to health workers on which health services are most beneficial. 


\begin{tabular}{lcc}
\hline Gender & & \\
Male & $31(65.96 \%)$ & $39(66.10 \%)$ \\
Female & $16(34.04 \%)$ & $20(33.90 \%)$ \\
Age (years) & 44 & 42 \\
Median & $25-68$ & $22-64$ \\
Range & & \\
Follow-up (months) & 64 & 50 \\
Median & $44-89$ & $38-61$ \\
Range & $11(23.40 \%)$ & $10(16.95 \%)$ \\
AJCC stage & $15(31.91 \%)$ & $9(15.25 \%)$ \\
T1N1M0 & $21(44.69 \%)$ & $40(67.80 \%)$ \\
T2N0M0 & & $37(62.71 \%)$ \\
T2N1M0 & $14(29.79 \%)$ & $22(37.29 \%)$ \\
Chemotherapy & $33(70.21 \%)$ & \\
Yes & & \\
No &
\end{tabular}

2D-CRT: two-dimensional conventional radiotherapy; IMRT: intensity-modulated radiotherapy.

\section{RESULTS}

\section{Patient characteristics}

Of 106 stage II NPC patients, 47 received 2D-CRT and 59 received IMRT. Disease-free survival of all subjects was more than 3 years. Table 1 summarizes patients' characteristics.

\section{QoL of IMRT versus 2D-CRT for the whole group}

In the whole group, IMRT $(\mathrm{n}=59)$ had higher mean scores in head and neck related symptoms and broad aspects of QoL for patients with stage II NPC than 2D-CRT $(\mathrm{n}=47)$ (Table 2). Clinical superiority of IMRT for QoL was significant on all functional scales and most symptom scales upon clinical interpretation (difference in mean scores $\geq 10$ points).

\section{QoL of IMRT versus 2D-CRT without concurrent chemotherapy}

In the radiotherapy alone subgroup, IMRT $(\mathrm{n}=22)$ had better QoL outcomes than 2D-CRT ( $\mathrm{n}=33$ ), except on scales of nausea/emesis, diarrhea, sticky saliva, coughing, pain killers, feeding tube, weight loss, and weight gain.
Differences of most scales between the two groups were significant (Table 3).

\section{QoL of IMRT versus 2D-CRT with concurrent chemotherapy}

In the concurrent chemotherapy subgroup, IMRT $(n=37)$ had better QoL outcomes than 2D-CRT $(n=14)$, except for symptoms of nausea/emesis, dyspnea, constipation, sticky saliva, pain killers, and feeding tube. Differences of most scales between the two groups were significant (Table 4).

\section{DISCUSSION}

This study suggests that IMRT has better outcomes in both functional and symptom scales of EORTC QLQ-C30 compared to 2D-CRT with or without concurrent chemotherapy. The result indicates that IMRT should be provided to NPC patients, irrespective of a concomitant substantial increase in expenditures if resources permit.

We observed that 2D-CRT adversely affected patients with regard to symptom scales, global QoL, and functional scales compared to IMRT for the whole group. Differences of most functional and symptom scales were significant upon clinical interpretation. The result 
Table 2: Mean quality of life scores of 2D-CRT versus IMRT for the whole group

\begin{tabular}{|c|c|c|c|c|c|c|}
\hline \multirow{2}{*}{ Scales } & \multicolumn{2}{|c|}{ 2D-CRT $(n=47)$} & \multicolumn{2}{|c|}{ IMRT $(n=59)$} & \multirow{2}{*}{$\mathbf{t}$} & \multirow{2}{*}{$\mathbf{p}$} \\
\hline & Mean & SD & Mean & SD & & \\
\hline \multicolumn{7}{|l|}{ EORTC QLQ-C30 } \\
\hline Global quality of life & 65.07 & 16.08 & 81.21 & 15.59 & -4.939 & 0.000 \\
\hline Physical functioning & 75.74 & 19.33 & 92.20 & 13.43 & -4.792 & 0.000 \\
\hline Role functioning & 74.47 & 22.21 & 90.43 & 14.64 & -4.112 & 0.000 \\
\hline Emotional functioning & 67.38 & 26.74 & 89.01 & 15.65 & -4.786 & 0.000 \\
\hline Cognitive functioning & 60.99 & 29.34 & 87.59 & 13.22 & -5.666 & 0.000 \\
\hline Social functioning & 61.35 & 24.35 & 91.13 & 13.39 & -7.348 & 0.000 \\
\hline Fatigue & 34.28 & 22.20 & 12.53 & 17.89 & 5.230 & 0.000 \\
\hline Nausea/emesis & 4.26 & 8.84 & 1.06 & 4.12 & 2.244 & 0.028 \\
\hline Pain & 18.09 & 17.32 & 8.16 & 11.97 & 3.233 & 0.002 \\
\hline Dyspnea & 11.35 & 15.97 & 4.96 & 16.99 & 1.877 & 0.064 \\
\hline Insomnia & 38.30 & 25.99 & 19.15 & 24.81 & 3.654 & 0.000 \\
\hline Appetite loss & 15.60 & 18.19 & 1.42 & 9.72 & 4.714 & 0.000 \\
\hline Constipation & 7.09 & 18.31 & 3.55 & 17.35 & 0.964 & 0.338 \\
\hline Diarrhea & 9.22 & 17.99 & 2.84 & 9.40 & 2.155 & 0.035 \\
\hline Financial problems & 44.68 & 27.17 & 21.28 & 24.50 & 4.386 & 0.000 \\
\hline \multicolumn{7}{|l|}{ EORTC QLQ-H\&N35 } \\
\hline Pain & 11.52 & 12.35 & 3.37 & 5.40 & 4.147 & 0.000 \\
\hline Swallowing & 25.71 & 17.62 & 5.32 & 8.93 & 7.076 & 0.000 \\
\hline Senses & 25.18 & 17.68 & 11.70 & 14.29 & 4.064 & 0.000 \\
\hline Speech & 10.64 & 11.57 & 2.84 & 7.13 & 3.934 & 0.000 \\
\hline Social contact & 28.90 & 22.51 & 4.79 & 10.95 & 6.603 & 0.000 \\
\hline Social eating & 12.77 & 12.10 & 2.55 & 6.60 & 5.078 & 0.000 \\
\hline Sexuality & 54.96 & 32.02 & 20.92 & 20.70 & 6.121 & 0.000 \\
\hline Teeth & 44.68 & 29.71 & 13.48 & 19.24 & 6.044 & 0.000 \\
\hline Opening mouth & 29.08 & 23.69 & 7.09 & 13.79 & 5.499 & 0.000 \\
\hline Dry mouth & 58.16 & 22.49 & 22.70 & 25.16 & 7.204 & 0.000 \\
\hline Sticky saliva & 9.93 & 18.28 & 4.26 & 16.47 & 1.581 & 0.117 \\
\hline Coughing & 13.48 & 17.94 & 12.06 & 17.62 & 0.387 & 0.700 \\
\hline Feeling ill & 22.70 & 20.97 & 8.51 & 17.68 & 3.545 & 0.001 \\
\hline Pain killers & 2.84 & 9.40 & 2.84 & 9.40 & 0.000 & 1.000 \\
\hline Nutritional supplements & 23.40 & 18.28 & 12.06 & 16.19 & 3.186 & 0.002 \\
\hline Feeding tube & 0.00 & 0.00 & 0.00 & 0.00 & 0.000 & 1.000 \\
\hline Weight loss & 5.67 & 12.66 & 1.42 & 6.80 & 2.030 & 0.046 \\
\hline Weight gain & 2.13 & 8.24 & 7.80 & 14.27 & -2.361 & 0.021 \\
\hline
\end{tabular}

2D-CRT: two-dimensional conventional radiotherapy; IMRT: intensity-modulated radiotherapy; SD: standard deviation; EORTC QOL-C30: European Organization for Research and Treatment of Cancer Quality of Life Questionnaire-Core 30; EORTC QOL-H\&N35: The EOTRC Quality of Life Questionnaire-Head and Neck 35. 
Table 3: Mean quality of life scores of 2D-CRT versus IMRT without concurrent chemotherapy

\begin{tabular}{|c|c|c|c|c|c|c|}
\hline \multirow{2}{*}{ Scales } & \multicolumn{2}{|c|}{ 2D-CRT $(n=33)$} & \multicolumn{2}{|c|}{ IMRT $(n=22)$} & \multirow{2}{*}{$\mathbf{t}$} & \multirow{2}{*}{$\mathbf{p}$} \\
\hline & Mean & SD & Mean & SD & & \\
\hline \multicolumn{7}{|l|}{ EORTC QLQ-C30 } \\
\hline Global quality of life & 69.95 & 15.30 & 86.74 & 11.69 & -4.364 & 0.000 \\
\hline Physical functioning & 80.61 & 19.10 & 97.58 & 8.11 & -4.528 & 0.000 \\
\hline Role functioning & 80.81 & 20.46 & 98.48 & 4.90 & -4.761 & 0.000 \\
\hline Emotional functioning & 74.49 & 23.75 & 95.08 & 14.47 & -3.990 & 0.000 \\
\hline Cognitive functioning & 66.67 & 30.33 & 94.70 & 9.47 & -4.959 & 0.000 \\
\hline Social functioning & 66.67 & 24.65 & 96.97 & 6.58 & -6.712 & 0.000 \\
\hline Fatigue & 26.94 & 19.84 & 6.06 & 8.21 & 5.391 & 0.000 \\
\hline Nausea/emesis & 4.04 & 9.35 & 1.52 & 4.90 & 1.306 & 0.197 \\
\hline Pain & 13.64 & 17.90 & 5.30 & 7.95 & 2.350 & 0.023 \\
\hline Dyspnea & 9.09 & 15.08 & 1.52 & 7.11 & 2.500 & 0.016 \\
\hline Insomnia & 28.28 & 20.62 & 12.12 & 21.93 & 2.777 & 0.008 \\
\hline Appetite loss & 14.14 & 18.69 & 0.00 & 0.00 & 4.346 & 0.000 \\
\hline Constipation & 8.08 & 20.46 & 0.00 & 0.00 & 2.268 & 0.030 \\
\hline Diarrhea & 7.07 & 18.18 & 1.52 & 7.11 & 1.584 & 0.120 \\
\hline Financial problems & 39.39 & 28.20 & 9.09 & 15.19 & 5.152 & 0.000 \\
\hline \multicolumn{7}{|l|}{ EORTC QLQ-H\&N35 } \\
\hline Pain & 10.86 & 14.05 & 1.52 & 4.18 & 3.590 & 0.001 \\
\hline Swallowing & 22.22 & 18.00 & 1.89 & 5.10 & 6.129 & 0.000 \\
\hline Senses & 23.74 & 17.69 & 6.06 & 8.21 & 4.990 & 0.000 \\
\hline Speech & 9.43 & 11.82 & 1.52 & 3.90 & 3.564 & 0.001 \\
\hline Social contact & 24.24 & 22.57 & .38 & 1.78 & 6.046 & 0.000 \\
\hline Social eating & 12.12 & 11.72 & .30 & 1.42 & 5.730 & 0.000 \\
\hline Sexuality & 47.47 & 30.08 & 11.36 & 20.82 & 5.260 & 0.000 \\
\hline Teeth & 40.40 & 32.01 & 9.09 & 18.35 & 4.599 & 0.000 \\
\hline Opening mouth & 27.27 & 25.62 & 1.52 & 7.11 & 5.468 & 0.000 \\
\hline Dry mouth & 54.55 & 23.30 & 16.67 & 19.92 & 6.249 & 0.000 \\
\hline Sticky saliva & 7.07 & 16.15 & 1.52 & 7.11 & 1.739 & 0.089 \\
\hline Coughing & 10.10 & 17.65 & 10.61 & 18.93 & -0.101 & 0.920 \\
\hline Feeling ill & 18.18 & 20.57 & 6.06 & 16.70 & 2.400 & 0.020 \\
\hline Pain killers & 3.03 & 9.73 & 0.00 & 0.00 & 1.789 & 0.083 \\
\hline Nutritional supplements & 21.21 & 20.10 & 6.06 & 13.16 & 3.378 & 0.001 \\
\hline Feeding tube & 0.00 & 0.00 & 0.00 & 0.00 & 0.000 & 1.000 \\
\hline Weight loss & 2.02 & 8.08 & 1.52 & 7.11 & 0.238 & 0.813 \\
\hline Weight gain & 1.01 & 5.80 & 0.00 & 0.00 & 0.814 & 0.419 \\
\hline
\end{tabular}

2D-CRT: two-dimensional conventional radiotherapy; IMRT: intensity-modulated radiotherapy; SD: standard deviation; EORTC QOL-C30: European Organization for Research and Treatment of Cancer Quality of Life Questionnaire-Core 30; EORTC QOL-H\&N35: The EOTRC Quality of Life Questionnaire-Head and Neck 35. 
Table 4: Mean quality of life scores of 2D-CRT versus IMRT with concurrent chemotherapy

\begin{tabular}{|c|c|c|c|c|c|c|}
\hline \multirow{2}{*}{ Scales } & \multicolumn{2}{|c|}{ 2D-CRT (n=14) } & \multicolumn{2}{|c|}{ IMRT $(n=37)$} & \multirow{2}{*}{$\mathbf{t}$} & \multirow{2}{*}{$\mathbf{p}$} \\
\hline & Mean & SD & Mean & SD & & \\
\hline \multicolumn{7}{|l|}{ EORTC QLQ-C30 } \\
\hline Global quality of life & 53.57 & 11.65 & 73.20 & 15.48 & -4.294 & 0.000 \\
\hline Physical functioning & 64.29 & 14.93 & 86.31 & 13.96 & -4.933 & 0.000 \\
\hline Role functioning & 59.52 & 19.30 & 83.33 & 15.21 & -4.628 & 0.000 \\
\hline Emotional functioning & 50.60 & 26.65 & 79.95 & 18.37 & -4.480 & 0.000 \\
\hline Cognitive functioning & 47.62 & 22.51 & 77.48 & 15.82 & -5.334 & 0.000 \\
\hline Social functioning & 48.81 & 19.02 & 82.43 & 16.17 & -6.313 & 0.000 \\
\hline Fatigue & 51.59 & 17.76 & 20.12 & 19.92 & 5.178 & 0.000 \\
\hline Nausea/emesis & 4.76 & 7.81 & 1.35 & 6.06 & 1.474 & 0.157 \\
\hline Pain & 28.57 & 10.19 & 10.36 & 13.24 & 4.643 & 0.000 \\
\hline Dyspnea & 16.67 & 17.30 & 6.31 & 18.98 & 1.780 & 0.081 \\
\hline Insomnia & 61.90 & 22.10 & 24.32 & 23.11 & 5.243 & 0.000 \\
\hline Appetite loss & 19.05 & 17.12 & 2.70 & 12.12 & 3.276 & 0.004 \\
\hline Constipation & 4.76 & 12.10 & 4.50 & 19.50 & 0.046 & 0.964 \\
\hline Diarrhea & 14.29 & 17.12 & 2.70 & 9.22 & 2.403 & 0.029 \\
\hline Financial problems & 57.14 & 20.37 & 35.14 & 27.16 & 2.747 & 0.008 \\
\hline \multicolumn{7}{|l|}{ EORTC QLQ-H\&N35 } \\
\hline Pain & 13.10 & 7.10 & 6.31 & 6.92 & 3.106 & 0.003 \\
\hline Swallowing & 33.93 & 14.05 & 11.26 & 10.25 & 6.349 & 0.000 \\
\hline Senses & 28.57 & 17.82 & 13.06 & 15.28 & 3.090 & 0.003 \\
\hline Speech & 13.49 & 10.83 & 3.00 & 7.70 & 3.319 & 0.004 \\
\hline Social contact & 39.88 & 18.83 & 11.94 & 13.26 & 5.096 & 0.000 \\
\hline Social eating & 14.29 & 13.30 & 3.78 & 7.46 & 2.794 & 0.013 \\
\hline Sexuality & 72.62 & 30.39 & 32.43 & 21.14 & 5.350 & 0.000 \\
\hline Teeth & 54.76 & 21.11 & 23.42 & 22.03 & 4.583 & 0.000 \\
\hline Opening mouth & 33.33 & 18.49 & 15.32 & 21.65 & 2.959 & 0.006 \\
\hline Dry mouth & 66.67 & 18.49 & 28.83 & 25.05 & 5.135 & 0.000 \\
\hline Sticky saliva & 16.67 & 21.68 & 4.50 & 17.85 & 1.872 & 0.076 \\
\hline Coughing & 21.43 & 16.57 & 9.91 & 15.45 & 2.330 & 0.024 \\
\hline Feeling ill & 33.33 & 18.49 & 9.01 & 16.94 & 4.464 & 0.000 \\
\hline Pain killers & 2.38 & 8.91 & 3.60 & 10.49 & -0.386 & 0.701 \\
\hline Nutritional supplements & 28.57 & 12.10 & 16.22 & 16.89 & 2.898 & 0.007 \\
\hline Feeding tube & 0.00 & 0.00 & 0.00 & 0.00 & 0.000 & 1.000 \\
\hline Weight loss & 14.29 & 17.12 & .90 & 5.48 & 2.870 & 0.012 \\
\hline Weight gain & 4.76 & 12.10 & 14.41 & 16.74 & -2.273 & 0.030 \\
\hline
\end{tabular}

2D-CRT: two-dimensional conventional radiotherapy; IMRT: intensity-modulated radiotherapy; SD: standard deviation; EORTC QOL-C30: European Organization for Research and Treatment of Cancer Quality of Life Questionnaire-Core 30; EORTC QOL-H\&N35: The EOTRC Quality of Life Questionnaire-Head and Neck 35. 
was similar to previous studies [4-6]. However, patients included in the previous studies were mostly T3-4 or N2-3. Radiotherapy combined with chemotherapy is the primary treatment modality for advanced loco-regionally NPC. It was suggested that concurrent chemotherapy adversely affected QoL of NPC patients [7]. None of these studies could totally exclude the interference of chemotherapy on QoL. In order to exclude the interference of chemotherapy, we conducted a subgroup analysis to compare the QoL of IMRT versus 2D-CRT without concurrent chemotherapy. The result revealed that IMRT alone significantly improved the QoL compared to 2D-CRT alone. Moreover, our subgroup result also suggested that IMRT had better QoL than 2D-CRT with concurrent chemotherapy.

It has been suggested that IMRT has significantly lower radiation-induced toxicity than 2D-CRT [13], but the change in the patient-reported xerostomia scores or QoL may be not statistically different between the two groups [10]. Possibilities to explain this inconsistency are as follow: (1) QoL assessment may contain questions that are not specific to RT-induced toxicities. (2) The criteria used to differentiate between grade 1 and grade 2 of QoL is rather vague and subjective. (3) Physician and patient bias may exist in an unblinded randomization setting. (4) Previous studies used a small sample size and a relatively shorter follow-up time. However, this study shows that IMRT has better QoL with or without concurrent chemotherapy in a longer follow-up time. The result further confirms that lower radiation-induced toxicities of IMRT may produce better QoL compared to 2D-CRT [9].

IMRT increases the expenses for NPC treatment and eventually increases the financial difficulties of individuals in developing countries such as China. Some studies found that financial difficulties adversely affected QoL $[14,15]$. Consequently, IMRT would adversely affect QoL. However, we found that patients receiving 2D-CRT suffered from greater financial difficulties than those receiving IMRT. The potential interpretation was that patients received 2D-CRT because of financial difficulties. Financial burden after treatment gave patients receiving 2D-CRT worse QoL, but the relationship between financial problems and QoL is still unclear. Further controlled studies should be performed to test the interference of financial difficulties on QoL.

The result of our study should be interpreted with caution. The EORTC QLQ-H\&N35 may have some limitations in assessment of QoL of NPC patients. NPC has different biological characteristics and treatment than other head and neck cancers. Xerostomia, deafness, otitis media, and symptoms from organs at risk injury after radiotherapy are the main symptoms in NPC survivors. Although the EORTC QLQ-H\&N35 is a specific questionnaire assessing the QoL of head and neck cancer, it does not deal with adverse radiation effects well enough.

Limitations of this study should be considered: (1) The small sample size (106 patients) may lead to statistical error. (2) This study assessed the QoL at only one time point. A more methodologically sound approach would employ a longitudinal design in which the same individuals are assessed repeatedly at various time points.

In conclusion, this study suggests that IMRT improves most general aspects of QoL for patients with stage II NPC compared to 2D-RCT. IMRT is a better treatment technique for stage II NPC.

\section{MATERIALS AND METHODS}

\section{Patients}

We analyzed QoL data of patients with stage II NPC in the Cancer Hospital of Guangxi Medical University from June 2008 to June 2013. Inclusion criteria were (1) pathologically proven NPC, (2) stage II NPC per the 7th Edition of the UICC/AJCC staging system, (3) receiving radical radiotherapy or concurrent chemotherapy, and (4) disease-free survival $>3$ years. Exclusion criteria were (1) age $>70$ or $<18$ years, (2) recurrent or metastatic NPC, (3) receiving induced or adjuvant chemotherapy, (4) a second malignancy, except for cured skin basal cell carcinoma or early stage cervical cancer, (5) severe cerebral, cardiac, hematologic, renal, hepatic, or mental disease, or (6) an incomplete self-reporting questionnaire.

From June 2008 to June 2013, 235 patients with stage II NPC received radical treatment at the Cancer Hospital of Guangxi Medical University. There were 129 total excluded patients; 8 were lost to follow-up, 4 received induced chemotherapy, 40 received adjuvant chemotherapy, 5 died, 9 were loco-regional failures, 7 were distant failures, 51 were non-compliant, and 5 did not complete the questionnaire. This study finally included 106 patients treated with IMRT $(\mathrm{n}=59)$ or 2D-CRT $(\mathrm{n}=47)$.

\section{Radiotherapy}

Patients received 2D-CRT in two phases. In the first phase, patients were irradiated by 6-megavolt bilateral and opposing photon beams. The dose for the faciocervical field and lower anterior cervical field was 36 Gy. In the second phase, the dose for primary tumor was boosted from $66 \mathrm{~Gy}$ to $70 \mathrm{~Gy}$. The prescribed irradiation dose was 2 Gy per fraction with 5 daily fractions per week.

Patients received IMRT per the International Commission on Radiation Units and Measurements Report 62 guidelines. Gross tumor volume (GTVnx) and cervical lymph node tumor volume (GTVnd) were determined by CT/MRI. Clinical target volume (CTV) included the GTV with a $1-\mathrm{cm}$ to $1.5-\mathrm{cm}$ margin, the entire nasopharyngeal space, and the positive lymph node regions. The prescribed radiation dose was 66 Gy to 70.06 Gy in 30 to 31 fractions for GTV, and 54 Gy to 60 Gy in 30 fractions for CTV with 5 daily fractions per week. 


\section{Chemotherapy}

Patients received concurrent chemotherapy on days 1,22 , and 43 during radiotherapy. The chemotherapy regimen was cisplatin $100 \mathrm{mg} / \mathrm{m}^{2} / \mathrm{d}$ by intravenous infusion. Chemotherapy was postponed or discontinued for patients who experienced serious toxicity and could not recover before the next schedule.

\section{QoL measurement}

Patients' QoL data were obtained by two clinicians from our department, both of whom received a uniform training. A subset of the patients was instructed to answer the questions during the visit to our clinic. Most patients were assessed by telephone. QoL data of all patients was analyzed by a third investigator. Consent was obtained from all patients included in the study.

QoL assessment used the Chinese version of the European Organization for Research and Treatment of Cancer Quality of Life Questionnaire-Core 30-questions (EORTC QLQ-C30) and the Head and Neck 35-questions (EORTC QLQ-H\&N35) [16-19]. The EORTC QLQ-C30 is a cancer-specific questionnaire containing a global QoL score, five functional scales, three symptom scales, and six single items. The QLQ-H\&N35 is a site-specific questionnaire assessing QoL of head-and-neck cancer patients. The QLQ-H\&N35 contains seven multiple-item and six single-item scales. The standard score of all scales ranges from 0 to 100. A high score for a global QoL or functional scale represents a high/healthy level of global QoL or functioning, whereas a high score for a symptom scale represents a symptom problem. QoL changes of $\geq 10$ points were considered clinically relevant [20,21].

\section{Statistical analysis}

Statistical analysis was performed using SPSS for Windows version 16.0 (SPSS Inc., Chicago, IL). The T-test was used to compare the mean scores of QoL between two groups. All significance tests were two-sided and $\mathrm{P}$ value $<0.05$ was considered statistically significant.

\section{Ethics statement}

This cross-sectional study was approved by the Ethics Committee of Cancer Hospital of Guangxi Medical University.

\section{Abbreviations}

NPC: nasopharyngeal carcinoma; 2D-CRT: twodimensional conventional radiotherapy; IMRT: intensitymodulated radiotherapy; QoL: quality of life; GTV: gross tumor volume; CTV: clinical target volume; EORTC QOL-C30: European Organization for Research and Treatment of Cancer; Quality of Life Questionnaire-Core
30; EORTC QOL-H\&N35: The EOTRC Quality of Life Questionnaire-Head and Neck 35.

\section{Author contributions}

PXB and ZXD contributed to the conception of the study; HST and CKH contributed to manuscript preparation; JYM and MJL performed the data analyses; QS, LL, and CL helped perform the analysis with constructive discussions.

\section{CONFLICTS OF INTEREST}

The authors declare no conflicts of interest.

\section{FUNDING}

This study was supported by the Basic Ability Improvement Project for Young and Middle-aged Teachers of Guangxi Zhuang Autonomous Region 2016 (No. KY2016LX029) and the Research and Development Project of Guangxi Medical and Health Appropriate Technology (No. S201514).

\section{REFERENCES}

1. Cao SM, Simons MJ, Qian CN. The prevalence and prevention of nasopharyngeal carcinoma in China. Chin J Cancer. 2011; 30:114-9.

2. Chen QY, Wen YF, Guo L, Liu H, Huang PY, Mo HY, Li NW, Xiang YQ, Luo DH, Qiu F, Sun R, Deng MQ, Chen $\mathrm{MY}$, et al. Concurrent chemoradiotherapy vs radiotherapy alone in stage II nasopharyngeal carcinoma: phase III randomized trial. J Natl Cancer Inst. 2011; 103:1761-70. doi: 10.1093/jnci/djr432.

3. Su SF, Han F, Zhao C, Chen CY, Xiao WW, Li JX, Lu TX. Long-term outcomes of early-stage nasopharyngeal carcinoma patients treated with intensity-modulated radiotherapy alone. Int J Radiat Oncol Biol Phys. 2012; 82:327-33. doi: 10.1016/j.ijrobp.2010.09.011.

4. Huang TL, Chien CY, Tsai WL, Liao KC, Chou SY, Lin HC, Dean Luo S, Lee TF, Lee CH, Fang FM. Long-term late toxicities and quality of life for survivors of nasopharyngeal carcinoma treated with intensity-modulated radiotherapy versus non-intensity-modulated radiotherapy. Head Neck. 2016; 38:E1026-32. doi: 10.1002/hed.24150.

5. Song T, Fang M, Zhang XB, Zhang P, Xie RF, Wu SX. Sustained improvement of quality of life for nasopharyngeal carcinoma treated by intensity modulated radiation therapy in long-term survivors. Int J Clin Exp Med. 2015; 8:5658-66.

6. Fang FM, Tsai WL, Chen HC, Hsu HC, Hsiung CY, Chien CY, Ko SF. Intensity-modulated or conformal radiotherapy improves the quality of life of patients with nasopharyngeal 
carcinoma: comparisons of four radiotherapy techniques. Cancer. 2007; 109:313-21. doi: 10.1002/cncr.22396.

7. Cengiz M, Ozyar E, Esassolak M, Altun M, Akmansu M, Sen M, Uzel O, Yavuz A, Dalmaz G, Uzal C, Hicsonmez A, Sarihan S, Kaplan B, et al. Assessment of quality of life of nasopharyngeal carcinoma patients with EORTC QLQ-C30 and H\&N-35 modules. Int J Radiat Oncol Biol Phys. 2005; 63:1347-53. doi: 10.1016/j.ijrobp.2005.05.057.

8. Pan XB, Huang ST, Chen KH, Jiang YM, Ma JL, Qu S, Li L, Chen L, Zhu XD. Concurrent chemoradiotherapy degrades the quality of life of patients with stage II nasopharyngeal carcinoma as compared to radiotherapy. Oncotarget. 2017; 8:14029-38. doi: 10.18632/oncotarget.14932.

9. Pow EH, Kwong DL, McMillan AS, Wong MC, Sham JS, Leung LH, Leung WK. Xerostomia and quality of life after intensity-modulated radiotherapy vs. conventional radiotherapy for early-stage nasopharyngeal carcinoma: initial report on a randomized controlled clinical trial. Int J Radiat Oncol Biol Phys. 2006; 66:981-91. doi: 10.1016/j. ijrobp.2006.06.013.

10. Kam MK, Leung SF, Zee B, Chau RM, Suen JJ, Mo F, Lai M, Ho R, Cheung KY, Yu BK, Chiu SK, Choi PH, Teo PM, et al. Prospective randomized study of intensity-modulated radiotherapy on salivary gland function in early-stage nasopharyngeal carcinoma patients. J Clin Oncol. 2007; 25:4873-9. doi: 10.1200/JCO.2007.11.5501.

11. Co J, Mejia MB, Dizon JM. Evidence on effectiveness of intensity-modulated radiotherapy versus 2-dimensional radiotherapy in the treatment of nasopharyngeal carcinoma: meta-analysis and a systematic review of the literature. Head Neck. 2016; 38:E2130-42. doi: 10.1002/hed.23977.

12. Marta GN, Silva V, de Andrade Carvalho H, de Arruda FF, Hanna SA, Gadia R, da Silva JL, Correa SF, Vita Abreu CE, Riera R. Intensity-modulated radiation therapy for head and neck cancer: systematic review and metaanalysis. Radiother Oncol. 2014; 110:9-15. doi: 10.1016/j. radonc.2013.11.010.

13. Peng G, Wang T, Yang KY, Zhang S, Zhang T, Li Q, Han J, Wu G. A prospective, randomized study comparing outcomes and toxicities of intensity-modulated radiotherapy vs. conventional two-dimensional radiotherapy for the treatment of nasopharyngeal carcinoma. Radiother Oncol. 2012; 104:286-93. doi: 10.1016/j.radonc.2012.08.013.
14. Yu CL, Fielding R, Chan CL, Sham JS. Chinese nasopharyngeal carcinoma patients treated with radiotherapy: association between satisfaction with information provided and quality of life. Cancer. 2001; 92:2126-35. doi: 10.1002/cncr.1554.

15. Fang FM, Chiu HC, Kuo WR, Wang CJ, Leung SW, Chen HC, Sun LM, Hsu HC. Health-related quality of life for nasopharyngeal carcinoma patients with cancer-free survival after treatment. Int J Radiat Oncol Biol Phys. 2002; 53:959-68.

16. Bjordal K, de Graeff A, Fayers PM, Hammerlid E, van Pottelsberghe C, Curran D, Ahlner-Elmqvist M, Maher EJ, Meyza JW, Bredart A, Soderholm AL, Arraras JJ, Feine JS, et al. A 12 country field study of the EORTC QLQ$\mathrm{C} 30$ (version 3.0) and the head and neck cancer specific module (EORTC QLQ-H\&N35) in head and neck patients. EORTC Quality of Life Group. Eur J Cancer. 2000; 36:1796-807.

17. Zhao H, Kanda K. Testing psychometric properties of the standard Chinese version of the European Organization for Research and Treatment of Cancer Quality of Life Core Questionnaire 30 (EORTC QLQ-C30). J Epidemiol. 2004; 14:193-203.

18. Bjordal K, Hammerlid E, Ahlner-Elmqvist M, de Graeff A, Boysen M, Evensen JF, Biorklund A, de Leeuw JR, Fayers PM, Jannert M, Westin T, Kaasa S. Quality of life in head and neck cancer patients: validation of the European Organization for Research and Treatment of Cancer Quality of Life Questionnaire-H\&N35. J Clin Oncol. 1999; 17:1008-19.

19. Aaronson NK, Ahmedzai S, Bergman B, Bullinger M, Cull A, Duez NJ, Filiberti A, Flechtner H, Fleishman SB, de Haes JC, Stein Kaasa, Marianne Klee, David Osoba, et al. The European Organization for Research and Treatment of Cancer QLQ-C30: a quality-of-life instrument for use in international clinical trials in oncology. J Natl Cancer Inst. 1993; 85:365-76.

20. King MT. The interpretation of scores from the EORTC quality of life questionnaire QLQ-C30. Qual Life Res. 1996; 5:555-67.

21. Osoba D, Rodrigues G, Myles J, Zee B, Pater J. Interpreting the significance of changes in health-related quality-of-life scores. J Clin Oncol. 1998; 16:139-44. 